Airway segmentation and analysis for the study of mouse models of lung disease using microCT

This article has been downloaded from IOPscience. Please scroll down to see the full text article. 2009 Phys. Med. Biol. 547009

(http://iopscience.iop.org/0031-9155/54/22/017)

View the table of contents for this issue, or go to the journal homepage for more

Download details:

IP Address: 159.237.12.65

The article was downloaded on 05/10/2010 at $11: 27$

Please note that terms and conditions apply. 


\title{
Airway segmentation and analysis for the study of mouse models of lung disease using micro-CT
}

\author{
X Artaechevarria ${ }^{1}$, D Pérez-Martín ${ }^{1}$, M Ceresa $^{1}$, G de Biurrun $^{2}$, \\ D Blanco $^{2}, \mathbf{L}$ M Montuenga ${ }^{2}$, B van Ginneken ${ }^{3}$, C Ortiz-de-Solorzano $^{1}$ \\ and A Muñoz-Barrutia ${ }^{1}$ \\ ${ }^{1}$ Cancer Imaging Laboratory, Center for Applied Medical Research, 31008 Pamplona, Spain \\ 2 Biomarkers Laboratory, Center for Applied Medical Research, University of Navarra, 31008 \\ Pamplona, Spain \\ ${ }^{3}$ Image Sciences Institute, 3584CX Utrecht, The Netherlands \\ E-mail: xabiarta@unav.es
}

Received 13 July 2009, in final form 14 October 2009

Published 4 November 2009

Online at stacks.iop.org/PMB/54/7009

\begin{abstract}
Animal models of lung disease are gaining importance in understanding the underlying mechanisms of diseases such as emphysema and lung cancer. Micro-CT allows in vivo imaging of these models, thus permitting the study of the progression of the disease or the effect of therapeutic drugs in longitudinal studies. Automated analysis of micro-CT images can be helpful to understand the physiology of diseased lungs, especially when combined with measurements of respiratory system input impedance. In this work, we present a fast and robust murine airway segmentation and reconstruction algorithm. The algorithm is based on a propagating fast marching wavefront that, as it grows, divides the tree into segments. We devised a number of specific rules to guarantee that the front propagates only inside the airways and to avoid leaking into the parenchyma. The algorithm was tested on normal mice, a mouse model of chronic inflammation and a mouse model of emphysema. A comparison with manual segmentations of two independent observers shows that the specificity and sensitivity values of our method are comparable to the inter-observer variability, and radius measurements of the mainstem bronchi reveal significant differences between healthy and diseased mice. Combining measurements of the automatically segmented airways with the parameters of the constant phase model provides extra information on how disease affects lung function.
\end{abstract}

\section{Introduction}

High-resolution micro-computed tomography (micro-CT) is an excellent tool to study rodent models of lung disease because of the inherent $\mathrm{x}$-ray absorption contrast between tissue and air 
that exists in the lungs (Ford et al 2007, Johnson 2007). This technology has been successfully used to study different disease models such as lung cancer and emphysema (De Clerck et al 2004, Postnov et al 2005, Froese et al 2007). Furthermore, its applications are likely to grow with the advent and improvement of flat-panel detectors.

Latest research on lung cancer points toward common initiation mechanisms in lung cancer, chronic obstructive pulmonary disease (COPD) and inflammatory lung disease (de Torres et al 2007, Houghton et al 2008, Cassidy et al 2007). In this scenario, the analysis of biologically relevant small animal models will be critical to gain knowledge on the specifics of each disease and the relationships existing between them. The widespread use of micro-CT in lung disease-related animal studies raises the need for precise and robust automated analysis and quantification tools for micro-CT images. Standardized automated techniques are thus required to allow for interlaboratory comparisons and reproducible validation of the results.

Segmenting the airways is required by most automatic lung analysis algorithms, especially in mice micro-CT images, due to the relatively large volume occupied by the airways. It is well known that the functionality of central airways and lung peripheral areas varies considerably, and the morphological effects of a great number of lung pathologies are different in the airways and the parenchyma. Separating airways from the rest of the lung is thus of the utmost importance when analyzing a disease (such as emphysema, chronic inflammation or cancer) which affects lung density differentially. Moreover, the analysis of segmented airways may be interesting in itself to study airway-specific morphological changes such as stenosis, bronchiectasis, etc. Finally, the airways can be used as a reference for image registration in follow-up studies or for atlas-based segmentation of the lungs, lobes and pulmonary segments.

To the best of our knowledge, only two airway segmentation methods in micro-CT images have been reported that are of use in mice. Chaturvedi and Lee (2005) segmented silicon casts of excised mice lungs using an interactive region growing algorithm. More relevant to the present work, Shi et al (2007) reported a fully automatic algorithm to segment in vivo images of healthy mice, acquired with an iso-pressure breath hold protocol (Namati et al 2006). This algorithm works by searching airway lumens in 2D transversal slices that are then used to reconstruct the full 3D tree. The main drawback of this method is that it is based on the search of candidates in 2D, which may lead to problems due to the high variability of the shape of the airway lumens as seen in transversal slices. Moreover, reported computation times were fairly high (about $30 \mathrm{~min}$ for each scan).

Due to scarce previous work on mouse airway segmentation, it is worth reviewing the most relevant approaches that exist for segmenting the human airways in CT images. Schlathoelter et al (2002) introduced an algorithm for simultaneous segmentation and reconstruction of the airways. This algorithm was based on a propagating front that divides the tree into branches during segmentation. The framework was extended and generalized in a subsequent work by Bülow et al (2004). More recently, van Ginneken et al (2008) also used the framework for human airway segmentation with a multi-threshold approach to increase robustness. Kiraly et al (2002) compared two different methods: an adaptive region growing algorithm and an algorithm that combined region growing and mathematical morphology. Their conclusion was that the region growing method was faster than the hybrid method, but also slightly less accurate. Aykac et al (2003) used a two-step approach. In a first step, candidate airways were identified on transversal slices using grayscale morphological reconstruction. In a second step, valid candidates were connected to build a 3D airway tree. Fetita et al (2004) also employed mathematical morphology to obtain a first approximation of the airways. The morphological operator worked in 3D and was specifically devised for this purpose. An energy-minimizing reconstruction algorithm was used to build the final airway tree. The work by Tschirren et al (2005) was based on fuzzy connectivity. They made use of small adaptive regions of interest 
around the already segmented airway areas. Thus, the algorithm adapted to local image characteristics, leaks were detected early and the computing time was reduced. In a recent work, Graham et al (2008) present a method that can be summarized in three main steps. In the first step, a conservative segmentation of the major airways is obtained via 3D region growing on heavily smoothed data. Then, possible branch segments are detected and connected to each other by nonlinear filtering and surface interpolation. The final segmentation is obtained using a global graph partitioning algorithm, which connects the valid branch segments to the major airways.

The main reason that prevents a direct translation of these algorithms to mice micro-CT data is the low signal-to-noise ratio (SNR) of the images, caused by the required small voxel size and the limitation on radiation dose imposed by the in vivo studies. In particular, following the simple model assumptions from Ford et al (2003), a reduction of one order of magnitude in voxel size $(0.5 \mathrm{~mm}$ in CT to $50 \mu \mathrm{m}$ in micro-CT) implies an increase of two orders of magnitude in the variance of the linear attenuation coefficient, if the rest of parameters remain unchanged.

Taking this into account, we decided to develop the flexible segmentation and reconstruction framework first reported by Schlathoelter et al (2002). This framework has multiple advantages. First, it allows for simultaneous segmentation and reconstruction of treelike structures. Second, the topological and morphological information from the segmented tree can be used to guide the segmentation of the remaining branches. Third, its modular configuration allows for easy introduction of application-specific segmentation rules. In fact, one of the major contributions of our work is the use of new features when compared to those in the previously reported applications of the framework, to adapt to the low SNR and the special morphology of the mice airways.

To complete the morphological information provided by the image-based measurements, we use respiratory system input impedance measurements and the constant phase model parameters, which have been widely used to assess respiratory mechanics in multiple animal models (Hantos et al 1992, Collins et al 2003, Tomioka et al 2002). The combination of microCT imaging and constant phase model parameters has also been used to analyze animal models of lung disease before. In particular, Lundblad et al (2007) qualitatively analyzed ex vivo microCT images in a mouse model of allergical inflammation and combined it with measurements of tissue elastance. In this work, we propose to use quantitative airway measurements since this approach is likely to provide valuable information to better understand the morphology and function of lungs affected by diseases such as emphysema and inflammation.

The rest of the article is structured as follows. In section 2, we briefly summarize the airway segmentation framework and give details about the new features we have introduced. In section 3, the image acquisition protocol, which includes the respiratory system input impedance measurement, and the airway segmentation validation experiments are detailed. Results are presented in section 4. A final discussion (section 5) concludes the article.

\section{Methods}

\subsection{Prefiltering}

Micro-CT images contain high levels of noise. This justifies the need for a filtering step before the analysis. To this end, we used a 3D grayscale closing by reconstruction filter with a spherical structuring element of radius 1 voxel (Vincent 1993). The 2D version of this filter was reported to yield positive results in the previous work by Shi et al (2007). This filter increases the contrast of the darkest regions of the image while preserving the shape of the 


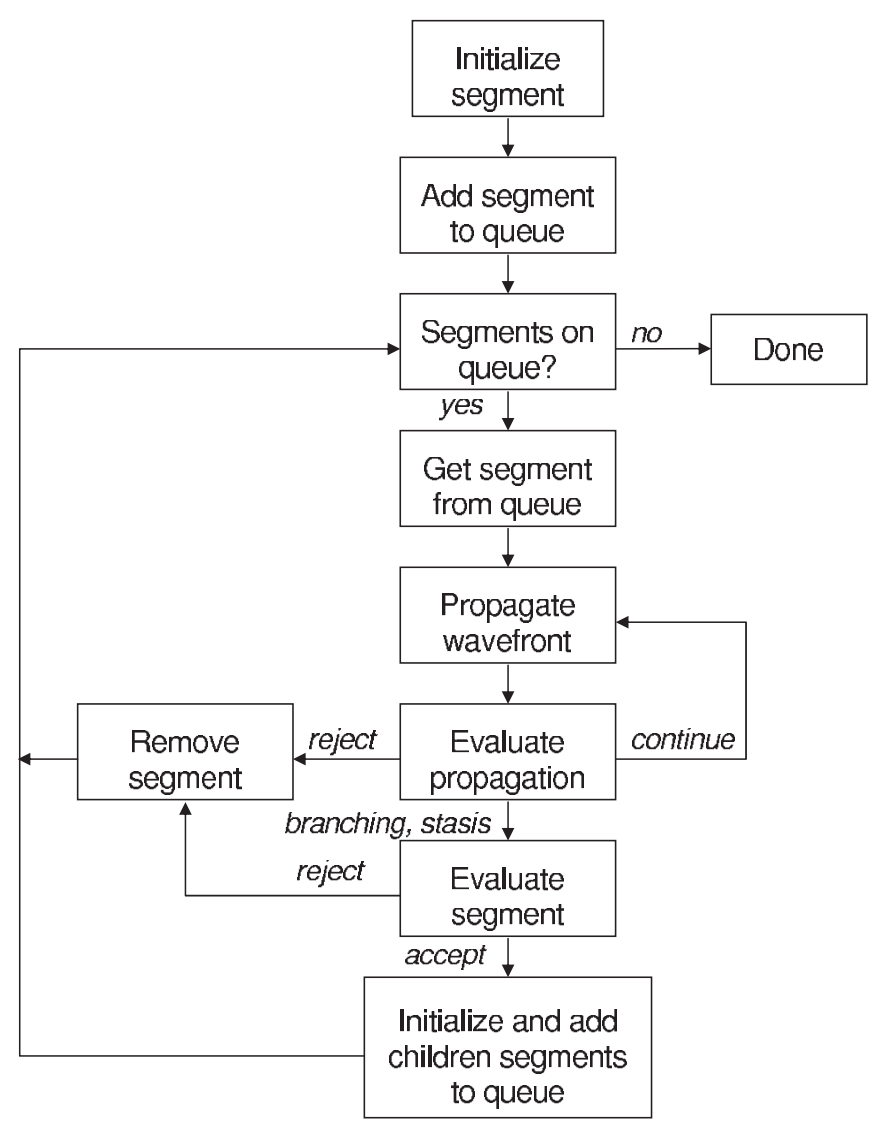

Figure 1. Block diagram of general tree segmentation and reconstruction framework.

structures. The radius was selected because it represented a good trade-off between noise removal and contour information preservation.

\subsection{Airway tree segmentation and reconstruction}

The adopted framework has been explained in detail in previous works by Schlathoelter et al (2002) and Bülow et al (2004). A block diagram summarizing the main execution flow is shown in figure 1. A key concept is the segment. A segment is a set of contiguous points that has been segmented by a growing wavefront without bifurcations. The growing wavefront is initialized in one seed point and grows into neighboring voxels that fulfill certain voxel acceptance criteria. After every wavefront propagation step, several conditions are checked to prevent leaks. When the wavefront bifurcates, new segments are initialized and added to the rest of pending segments in a segment queue. This queue is a first-in, first-out (FIFO) list, thus ensuring that branches from upper airways are processed first. When a segment is finished, its correctness is checked in a segment evaluation step.

It was necessary to develop new voxel acceptance, propagation evaluation and segment evaluation criteria, due to the particularities of our segmentation task. Details about these new features are given in this subsection. 
2.2.1. Initialization. The initial wavefront consists of a seed point located inside the trachea detected on the first transversal slice. To find the trachea, the first image slice is automatically thresholded using the method described by $\mathrm{Hu}$ et al (2001). This threshold value separates air from tissue and it is iteratively computed for each image. Once this separation is done, two main objects appear in the thresholded binary image: the trachea and the background around the animal body. Other smaller objects can appear, for instance between the animal body and the bed. To select the trachea, the following process is used. In a first step, very small objects - smaller than 100 pixels - are removed. Next, very large objects-larger than 5000 pixels - are discarded. This step removes the background. The last step consists in computing the center of mass of the remaining objects. The most centered object is considered to be the trachea. The center of mass of the trachea in the first slice region is set as the initial seed for the wavefront propagation. The Insight Toolkit (Ibanez et al 2003) was used to implement the initialization and also the rest of steps of the algorithm.

2.2.2. Wavefront propagation. To propagate the wavefront, we use a $3 \mathrm{D}$ fast marching level set algorithm. In particular, our implementation is based on the algorithm described by Deschamps and Cohen (2001) and we refer to their work for details. An important aspect is that, since the intensity threshold is variable - as it will be detailed in subsection 2.2.3 - it is not guaranteed that the potential will be monotonically increasing as the wavefront propagates. Since the fast marching algorithm assumes this, the wavefront stops as soon as a negative growth of the potential is detected. An important advantage of a fast marching front compared to region growing is the dome shape of the wavefront, which is very helpful for correctly detecting bifurcations.

Bifurcation detection is a critical aspect of wavefront propagation. To detect a bifurcation, the connectivity of the wavefront is checked after every propagation step. If the wavefront is divided in two or more parts, a bifurcation exists. Sensitivity to detect a bifurcation depends on the connectivity size parameter. The connectivity size defines how many voxels around a given voxels are considered as neighbors. A neighbor connectivity of radius 1 means that only the 26 voxels around a given voxel are considered neighbors. If this size is increased to 2 , all voxels in the $5 \times 5 \times 5$ cube around a given voxel will be considered neighbors. Therefore, a low connectivity is very sensitive to bifurcations and will detect them immediately, although it is also sensitive to noise and will probably detect non-existent bifurcations caused by noise. To combine the advantages of high and low connectivities, we have implemented a variable connectivity scheme. Namely, a high connectivity size is used by default-cubical neighborhood of radius 3-and when rapid growth of the wavefront in successive steps is detected, the parameter is reduced to radius 1 , in order to detect the bifurcation more rapidly. The connectivity returns to the default value of 3 once the segment stops growing.

2.2.3. Voxel acceptance criteria. The viscosity function of the fast marching algorithm determines whether a voxel is to be added to the current segment or not. If a certain voxel fulfills the acceptance criteria, its viscosity value is set to 1 . Otherwise it is set to infinity. There are two criteria that a voxel must fulfill before being accepted: the similar intensity condition and the low gradient condition. The similar intensity condition is given by a variable upper threshold, which accounts for the large variability in image intensities observable in micro-CT images. In other words, voxels with an intensity lower than the variable intensity threshold, $T_{i}$, fulfill the similar intensity condition, while the rest do not. In other words, the low SNR and the strong artifacts (beam hardening, circular artifacts) cause the airway branches to have different intensities depending on their location. Therefore, it becomes necessary to have a 
threshold that varies within the same image. We define the variable threshold considering the intensity distribution of the current segment as well as the intensities of its ancestor segments:

$$
T_{i}=\mu_{l}+\alpha \cdot \max \left(\sigma_{p}, \sigma_{g p}\right),
$$

where $\mu_{l}$ is the mean intensity of the current segment ( $l$ stands for local), $\alpha$ is a tunable parameter, and $\sigma_{p}$ and $\sigma_{g p}$ are the standard deviations of the intensity values of the voxels in the parent and grandparent segments of the current one, respectively. The largest of both is taken, to avoid very small $\sigma$-s in the case of a short or very homogeneous segment, which would lead to a too low threshold $T_{i}$. Intensities are averaged in a $3 \times 3 \times 3$ neighborhood around the voxel of interest, to minimize the effect of noise. In our experience with different imaging protocols, $\alpha$-values between 1.2 and 2 work generally well. If the image is very noisy $\alpha$ must be rather small (near 1.2), while less noisy images require an $\alpha$-value closer to 2 .

For the low gradient condition, the three-dimensional Sobel gradient is computed and voxels are rejected if their Sobel gradient value exceeds a given threshold $T_{s}$. This way segmentation leakage from the airways and into the lungs is avoided.

2.2.4. Propagation evaluation. There are two criteria to accept or reject a propagation step. The first one controls the current size of the wavefront and prevents it from growing in excess. This is implemented by not allowing wavefronts to be larger than the wavefronts in their parent segments. To account for noise and anatomical irregularities, the propagation stops if

$$
r>\gamma \cdot \frac{\min \left(r_{\mathrm{anc}}\right)+\operatorname{mean}\left(r_{\mathrm{anc}_{\min -r}}\right)}{2},
$$

where $r$ is the current wavefront radius, $\gamma$ is a tunable parameter that allows certain growth, $\min \left(r_{\mathrm{anc}}\right)$ is the minimum radius among all ancestors of the current segment and mean $\left(r_{\mathrm{anc}} \mathrm{min}_{\mathrm{m}}\right)$ is the mean value of wavefront radius of the ancestor containing the minimum radius. The latter is averaged with the smallest radius because the smallest radius alone resulted in a too noisy measure, which limited propagation in some cases. The value of $\gamma$ indicates the tolerance to the growth of the airway diameter as the wavefront propagates inside the tree. A large value allows segments to be wider than their ancestors, but can also lead to accept leaks as correct airways. Given that mouse airway segments can be considerably wider than their ancestors-especially the right mainstem bronchus can be wider than the trachea-the values between 1.5 and 2 should be considered.

The second propagation evaluation rule refers to the number of neighbor segments. This number is limited to 2 because the number of neighbors computed before the current segment has stopped its propagation and its children segments have been initialized. A number greater than 2 generally indicates that several small segments are growing next to each other, which is a common indicator of leakage.

2.2.5. Segment evaluation. Three different parameters are considered to accept or reject a fully grown segment. A segment is considered to be fully grown when a bifurcation of the wavefront is detected or it cannot grow further.

To evaluate if the wavefront has propagated uniformly, the growth rate (GR) is measured, which is given by

$$
\mathrm{GR}=\frac{1}{N} \sum_{i=1}^{N} \frac{\left|W_{i}\right|}{\left|W_{i-1}\right|},
$$

where $N$ is the number of propagation steps in the segment and $\left|W_{i}\right|$ is the number of voxels of the wavefront at propagation step $i$. Thus, a GR larger than 1 indicates that the segment 
has grown during propagation. A threshold $T_{\mathrm{GR}}$ slightly larger than 1 allows a slight growth of the segment.

Then, the discrete compactness $(C)$ is computed, as defined by Bribiesca (2008) for a solid volume of $n$ voxels in 3D:

$$
C=\frac{n-A / 6}{n-(\sqrt[3]{n})^{2}},
$$

where $A$ is the area of the enclosing surface of the segment, and corresponds to the sum of the areas of the external plane polygons of the voxels which form the visible faces of the solid. Correct airways tend to be more compact than leakages. A threshold $T_{C}$ is defined to separate correct and incorrect segments.

Finally, the difference between the sizes of the last and the first wavefront is also computed and compared to a threshold $T_{W}$ because a large difference is a typical sign of leakage.

These three parameters are combined through voting, as detailed in algorithm 1. Two of the three parameters must be above the thresholds in order to reject a segment.

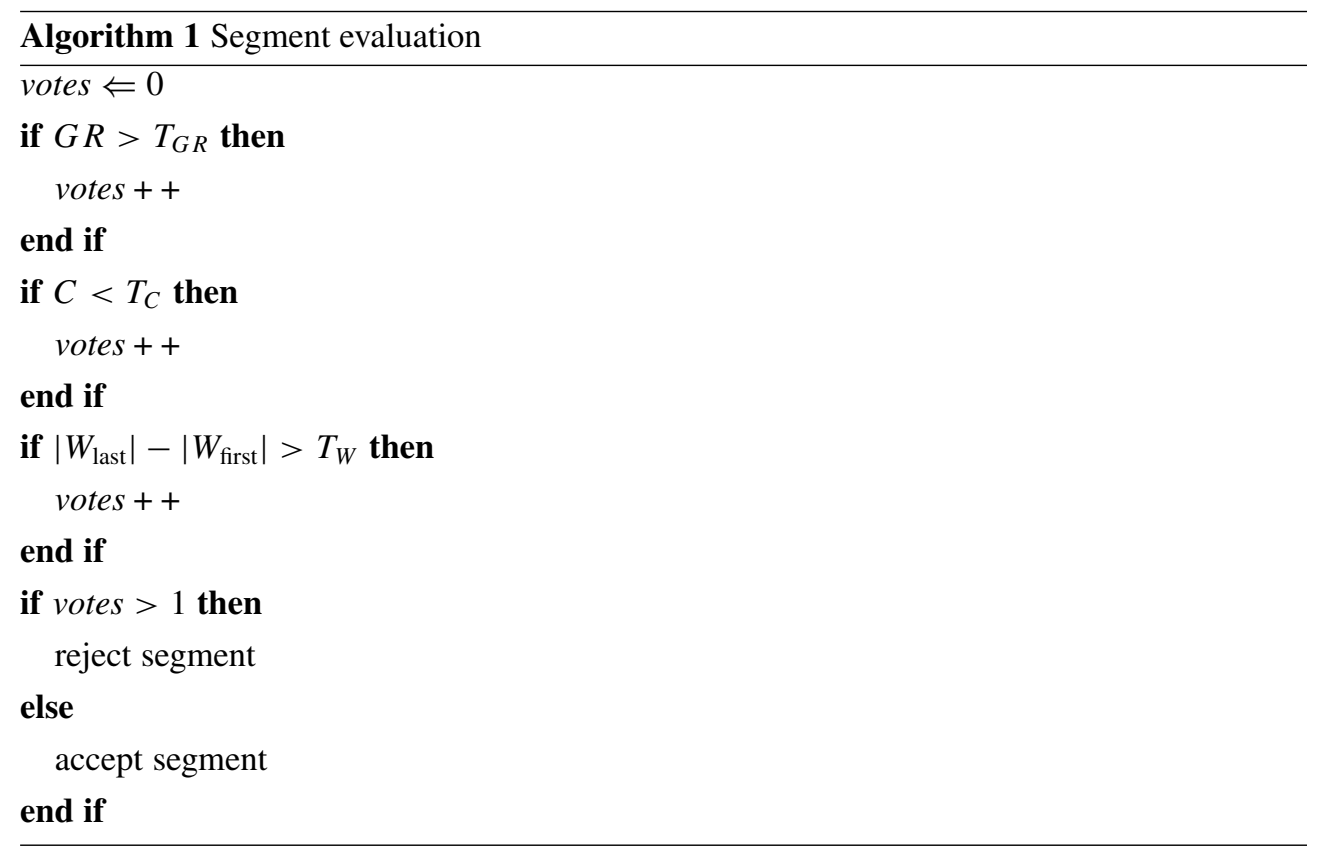

\section{Experiments}

\subsection{Image dataset}

We tested our algorithm on three different groups of $10 \mathrm{~A} / \mathrm{J}$ male mice each (Harlan UK Ltd, Oxon, UK), scanned at 12 weeks of age and with a mean weight of $21.1 \mathrm{~g}$ (variance 1.45). The three groups received a different treatment 4 weeks before the scan. Mice from the control group were intratracheally instilled with $90 \mu \mathrm{l}$ of saline. The second group was instilled with elastase in a dose of 6 Units per $30 \mathrm{~g}$ (EC134GI, EPC, MI, USA), which is known to cause severe emphysema in A/J mice (Valentine et al 1983). The third group was instilled with crystalline silica ( $9 \mathrm{mg}$ in $90 \mu \mathrm{l}$ saline per mice). The crystalline silica sample was $99 \%$ pure alpha-quartz (Min-U-Sil 5, US Silica Co., Berkeley Springs, WV, USA), with a particle size of $<5 \mu \mathrm{m}$. This single treatment causes silicosis which occurs with severe inflammation in 
the first weeks after instillation (Saffiotti et al 1996). All procedures were approved by the University of Navarra Institutional Animal Care and Use Committee, in compliance with the European Union relevant guidelines for the use of laboratory animals.

\subsection{Imaging protocol}

To ensure high-quality thoracic images free from movement-related artifacts, our protocol uses artificial ventilation, and the micro-CT projections are acquired during ventilator-induced breath holds.

In particular, the data acquisition-input impedance parameters and micro-CT imageprotocol is an adaptation of that by Namati et al (2006). Animals were anesthetized with an intraperitoneal injection of $90 \mathrm{mg} \mathrm{kg}^{-1}$ ketamine and $10 \mathrm{mg} \mathrm{kg}^{-1}$ xylacine. Endotracheal intubation was performed on anesthetized animals using the BioLite system (Biotex, Houston, TX, USA), to illuminate the trachea with a fiber optic stylet. After intubation, animals were connected to a Flexivent rodent ventilator (Scireq, Montreal, Canada) at a rate of 200 breaths $\mathrm{min}^{-1}$ and a tidal volume of $10 \mathrm{ml} \mathrm{kg}^{-1}$. Animals were kept breathing isoflurane at $2 \%$ concentration until complete relaxation was achieved. $0.5 \%$ isoflurane was maintained during the rest of the experiment. No paralyzer was required in order to prevent mice from trying to breath on their own. Once the animals were connected to the ventilator and did not show any reflex or attempt to breath on their own, the forced oscillation technique was employed to compute the airway resistance (Raw), airway inertance (I), tissue damping $(G)$ and tissue elastance $(H)$ (Hantos et al 1992). The manoeuvre was performed three times and the mean value for each parameter was calculated.

After obtaining the constant phase model parameters, the animals were scanned. 700 micro-CT projections were acquired during iso-pressure breath holds at $12 \mathrm{cmH}_{2} \mathrm{O}$, which represents a physiological pressure and minimizes the probability of ventilator-induced lung injury (VILI) (Dreyfuss and Saumon 1998). Breath hold duration was $650 \mathrm{~ms}$ and normal breathing was induced for two inspiration-expiration cycles between breath holds. A total lung capacity (TLC) perturbation was performed every 20 breath holds. This inflates the lung to the maximum capacity $\left(30 \mathrm{cmH}_{2} \mathrm{O}\right)$ for $3 \mathrm{~s}$ and contributes to prevent atelectasis (Allen et al 2006). No projections were acquired during the TLC perturbations.

Images were acquired with a Micro-CAT II scanner (Siemens Pre-Clinical Solutions, Knoxville, TN), with a source voltage of $80 \mathrm{kVp}$ and a current of $500 \mu \mathrm{A}$. The exposure time per projection was $450 \mathrm{~ms}$ and each projection was acquired during the $650 \mathrm{~ms}$ iso-pressure breath holds at peak inspiration $\left(12 \mathrm{~cm} \mathrm{H}_{2} \mathrm{O}\right)$. This resulted in images of 640 slices with $1024 \times$ 1024 voxels per slice, and an isotropic voxel size of $46 \mu \mathrm{m}$. A water phantom was used to calibrate the images to Hounsfield Units (HU). The resolution estimated by the Resolution Estimator software (Siemens Pre-Clinical Solutions, Knoxville, TN) was $43.74 \mu \mathrm{m}$. Scan time was approximately $30 \mathrm{~min}$ and the dosage computed by the Dose Calculator software (Siemens Pre-Clinical Solutions, Knoxville, TN) was 71.6 cGy per scan. To estimate image noise, we scanned a cylindrical phantom filled with water and computed the standard deviation of the water region (mean $0.97 \mathrm{HU}$ ), which resulted in $74.55 \mathrm{HU}$.

After the scan, animals were intraperitoneally injected with warm saline (10 $\mu \mathrm{l}$ per $1 \mathrm{~g})$ to aid recovery from anesthesia and artificial ventilation.

\subsection{Airway segmentation evaluation measures}

Automatic airway segmentations were compared to manual segmentations, which were taken as reference. Only every fifth transversal slice was considered for evaluation, due to the 
long time required for manual segmentations. The Amira software (Visage Imaging, Fürth, Germany) was used to perform the manual segmentations. To this end, a seed was placed inside every visible airway and 2D region growing was applied to segment the airway. The upper threshold for region growing was modified to adjust to local airway characteristics in each case. In occasions in which this approach failed (mainly due to leakage into the parenchyma), the airways were delineated manually. The entire manual delineation process took around 90 min per scan. To assess inter-rater variability, a second expert segmented five scans from each of the three groups.

To assess the accuracy of our segmentation method, we computed the true positive volume fraction (TPVF) and false positive volume fraction (FPVF) as defined in the work of Shi et al (2007):

$$
\mathrm{TPVF}=\frac{\left|S_{A} \cap S_{M}\right|}{\left|S_{M}\right|}, \quad \mathrm{FPVF}=\frac{\left|S_{A}-S_{M}\right|}{\left|S_{M}\right|},
$$

where $S_{A}$ is the automatic segmentation, $S_{M}$ is the manual segmentation, $\left|S_{A} \cap S_{M}\right|$ represents the cardinality of the intersection between the two segmentations and $\left|S_{A}-S_{M}\right|$ represents the number of pixels that were marked as airway by the automatic algorithm but not by the manual segmentation.

\subsection{Test parameter sets}

Our airway segmentation algorithm has a number of parameters that must be set. For this purpose, we used two extra images from each group, which were excluded from the validation study. The tunable parameters can be divided in two different groups: wavefront propagation and segment evaluation. The wavefront propagation parameters are $\alpha$ (equation (1)), $T_{s}$ and $\gamma$ (equation (2)). The parameter $\alpha$ (see subsection 2.2.2) acts on the threshold of the propagation: a high $\alpha$-value leads to a high threshold. $T_{s}$ is the threshold for the Sobel gradient and, similarly, the higher the threshold the less restrictive the propagation. The last parameter related to propagation is $\gamma$ (equation (2)). A smaller value implies more restrictions to radius growth in subsequent wavefronts. Three thresholds $\left(T_{\mathrm{GR}}, T_{C}\right.$ and $\left.T_{W}\right)$ comprise the segment evaluation parameters. A more lenient set generally leads to more accepted segments, with the risk of more invalid segments being accepted.

We used a simple methodology to set the tunable parameters. The wavefront propagation parameters were selected first, by visually observing the results in two images of each group. These parameters were set so that all the main bronchi and most of the small bronchi were segmented, without excessive leakage into the parenchyma. To select $T_{\mathrm{GR}}, T_{C}$ and $T_{W}$, the growth rate, compactness and difference between last and first wavefront parameters of a few correct and incorrect segments were observed, and thresholds that best separated those two groups were chosen.

Due to the very different image characteristics of the three groups, two different parameter sets resulted from the tuning process. The first set, aimed at control and silica-treated mice, has more lenient rules for both propagation and segment evaluation than the second set. The reason is that airway walls tend to be worse defined in elastase-treated animals and this results in a much higher leakage probability. Precise values for parameters are given in table 1 .

\subsection{Robustness to image noise}

To test how the algorithm behaves on images of different characteristics, we scanned three $\mathrm{A} / \mathrm{J}$ mice with three different $\mathrm{X}$-ray doses. Two mice were 11 months old and the third one was 


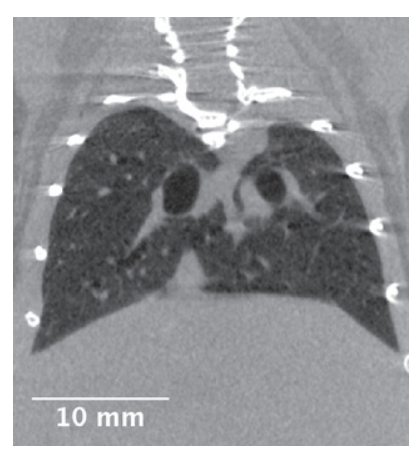

(a) Control

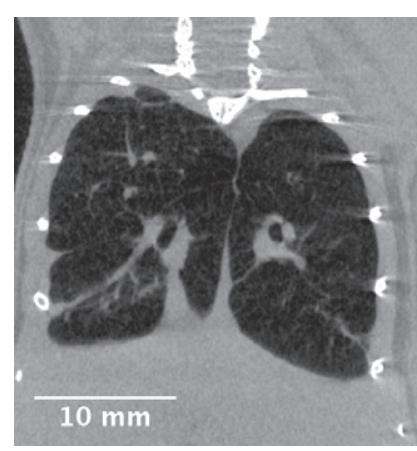

(b) Emphysema

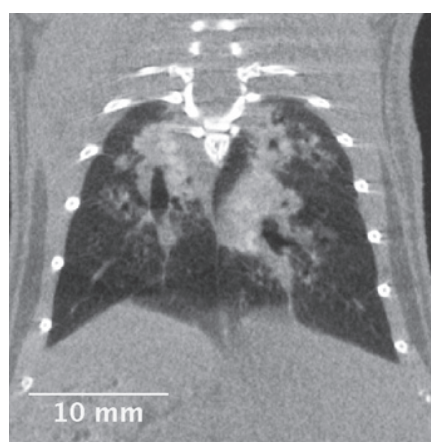

(c) Inflammation

Figure 2. Sample coronal micro-CT slices of control, emphysematous and inflammed mice lungs. Differences in lung texture and shape are clearly visible.

Table 1. Two test parameter sets, with different values for the tunable parameters of the airway segmentation and reconstruction algorithm. Values in set 1 were selected using two images of the control group and two images of the silica group. Two images of the elastase group were used for set 2 . The symbol $|S|$ indicates the number of voxels of the evaluated segment.

\begin{tabular}{lll}
\hline & Set 1 & Set 2 \\
\hline$\alpha$ & 1.4 & 1.3 \\
$T_{s}$ & none & 4250 \\
$\gamma$ & 2.5 & 2.5 \\
$T_{\mathrm{GR}}$ & 1.2 & 1.1 \\
$T_{C}$ & 0.7 & $5 \times 10^{-5} \cdot|S|$ \\
$T_{W_{d}}$ & none & $3.5 \times 10^{-2} \cdot|S|$ \\
\hline
\end{tabular}

16 months old. The imaging protocol was in all cases virtually identical to the one described in subsection 3.2. The only difference was the tube voltage, which was set to $80 k V_{p}$ for the normal dose images, to $65 k V_{p}$ for the low dose images and to $40 k V_{p}$ for the very low dose images. The standard deviation of the water volume in a water phantom used to estimate the noise was $78.32 \mathrm{HU}, 86.64 \mathrm{HU}$ and $94.42 \mathrm{HU}$ respectively.

\section{Results}

Micro-CT sample images from the three groups are shown in figure 2. Compared to the control group, emphysematous mice show considerably darker lungs, due to the loss of parenchymal tissue (Froese et al 2007). This, together with a stronger tendency to movement-related artifacts, leads to poor intensity contrast between airways and parenchyma. The airway diameter is also generally smaller and lungs appear more inflated. In contrast, the silica group shows large inflammed central areas which display with high intensity in the image. Some lung lobes and airways leading to them can appear collapsed.

Table 2 shows the segmentation accuracy results for the three different groups using the two different parameter sets presented in table 1. As expected, the first parameter set yields good results in the control and silica groups, while a more restrictive set is required for the elastase-treated emphysema group. 
Table 2. Results of the automatic segmentation on the three different image datasets. Mean TPVF and FPVF are indicated, together with the standard deviation in parentheses. The parameter set for each group is marked in bold.

\begin{tabular}{llll}
\hline Dataset & Parameter set & TPVF $(\%)$ & FPVF $(\%)$ \\
\hline Control & 1 & $\mathbf{8 9 . 4 4}(\mathbf{3 . 2 6})$ & $\mathbf{7 . 9 7}(\mathbf{5 . 5 3})$ \\
& 2 & $73.14(22.88)$ & $2.82(2.18)$ \\
Elastase & 1 & $53.28(11.41)$ & $53.76(31.69)$ \\
& 2 & $\mathbf{8 1 . 2 0}(\mathbf{3 . 5 8})$ & $\mathbf{1 2 . 9 9}(\mathbf{8 . 1 7})$ \\
Silica & 1 & $\mathbf{8 7 . 8 6}(\mathbf{3 . 8 4})$ & $\mathbf{6 . 5 2}(\mathbf{3 . 0 1})$ \\
& 2 & $72.04(21.97)$ & $2.98(2.10)$ \\
\hline
\end{tabular}

Table 3. Inter-observer variability. Mean TPVF and FPVF of the second observer's segmentations are indicated, together with the standard deviation in parentheses.

\begin{tabular}{lll}
\hline Dataset & TPVF(\%) & FPVF(\%) \\
\hline Control & $96.06(1.41)$ & $8.81(1.07)$ \\
Elastase & $95.59(0.86)$ & $14.3(1.82)$ \\
Silica & $90.16(4.36)$ & $6.76(3.51)$ \\
\hline
\end{tabular}

The result of manual segmentations depends up to a certain point on the subjectiveness of the manual segmenter. These effects can be partially quantified by looking at inter-observer variability. In this case, it was estimated by comparing the manual segmentations of two independent observers on five randomly chosen images of each group. The first observer's segmentations were taken as reference to compute the TPVF and FPVF of the second observer. Results are shown in table 3, where relatively high values of TPVF and FPVF can be observed. Visual comparison of the two observers' segmentations revealed that the second observer drew larger contours around the airway lumens, which explains the high FPVF values.

Most oversegmentation errors of our automatic method occurred due to late detection of leaks, as shown in the 3D rendering of figure 3. A more restrictive set of segment acceptance rules would reduce these kind of leaks, but it would also affect the TPVF, as already seen in table 2 .

Computation time varies from image to image depending on particular image characteristics (airway wall thickness, movement artifact, etc) and lies between 1 and $3 \mathrm{~min}$, with non-optimized $\mathrm{C}++$ code on an Intel Xeon $3.20 \mathrm{GHz}$ processor.

Our algorithm not only segments the airway tree, but also provides a reconstruction of the bronchial tree, by means of a list of segments hierarchically related. The length, volume and mean wavefront size of each segment are also given. The mean wavefront size can be seen as a surrogate of airway area since the front propagates in the normal direction to the airway lumen. Figure 4 shows three renderings of images of the three groups, with main segments displayed in different gray levels. It must be noted that segments do not necessarily correspond to airway branches because they only have algorithmic meaning, as explained in subsection 2.2.

To gain a better understanding of the effect of the diseases on the lung physiology, we first compared the radii of the two mainstem bronchi of the three different mice groups. To have a more accurate measurement and to have segments that correspond to real airway branches, the algorithm was run again on the already segmented trees. Table 4 shows the mean radii of the three different groups. It can be seen that, as expected, the right mainstem bronchus is larger 


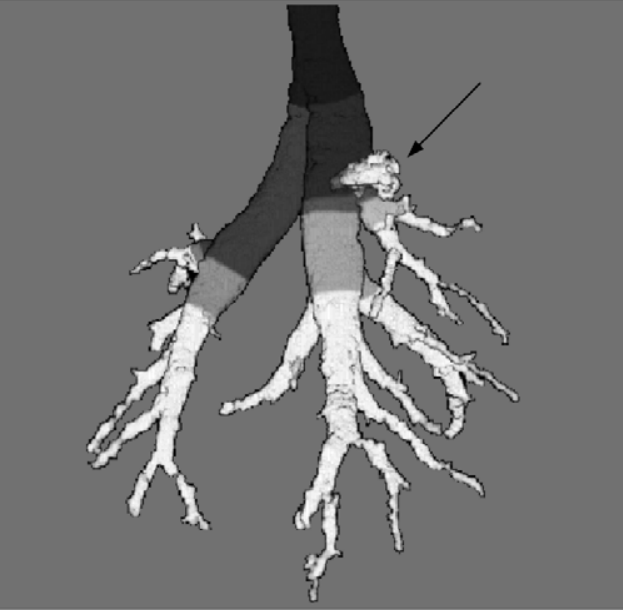

Figure 3. 3D rendering of a segmentation of a control mouse airway. The black arrow points at an undetected leakage in the upper-right side.

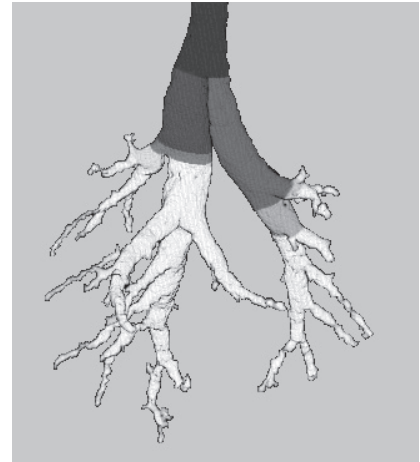

(a) Control

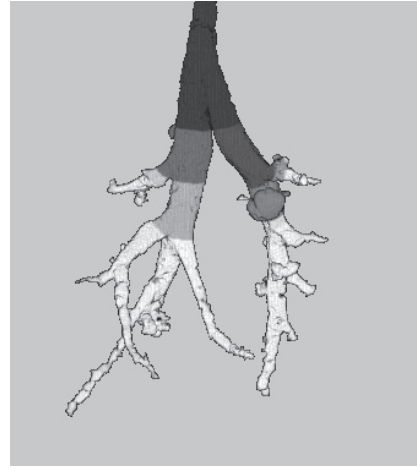

(b) Emphysema

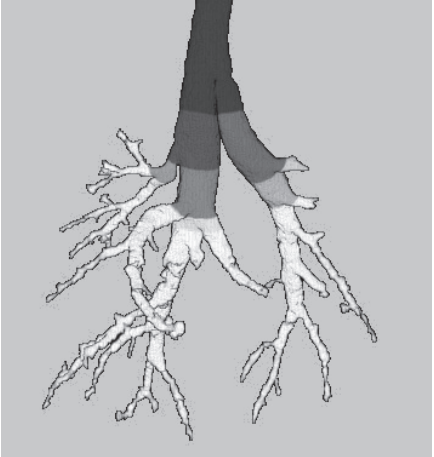

(c) Inflammation

Figure 4. Sample renderings of segmented airway trees of the three groups. In emphysema, less small branches are segmented due to the lack of contrast between airway and parenchyma in those areas, resulting in a less detailed segmentation compared to the other groups. A similar effect took place in manual segmentations. To allow a clear distinction between the main segments in the renderings, only main segments are shown in different gray levels, while smaller branches are all displayed in white.

Table 4. Mean radius of the right and left mainstem bronchus (MB) in $\mu \mathrm{m}$. (*) and (**) indicate $p<0.05$ and $p<0.01$ when compared to the control group using the Wilcoxon or Mann-Whitney $U$-test.

\begin{tabular}{lll}
\hline & Right MB & Left MB \\
\hline Control & 760.38 & 529.92 \\
Elastase & $613.64^{* *}$ & $475.18^{*}$ \\
Silica & $636.64^{* *}$ & $449.88^{*}$ \\
\hline
\end{tabular}

than the left and that the elastase and silica groups have smaller radii than the control group. The difference was statistically significant according to the Wilcoxon or Mann-Whitney $U$-test (Wilcoxon 1945). 


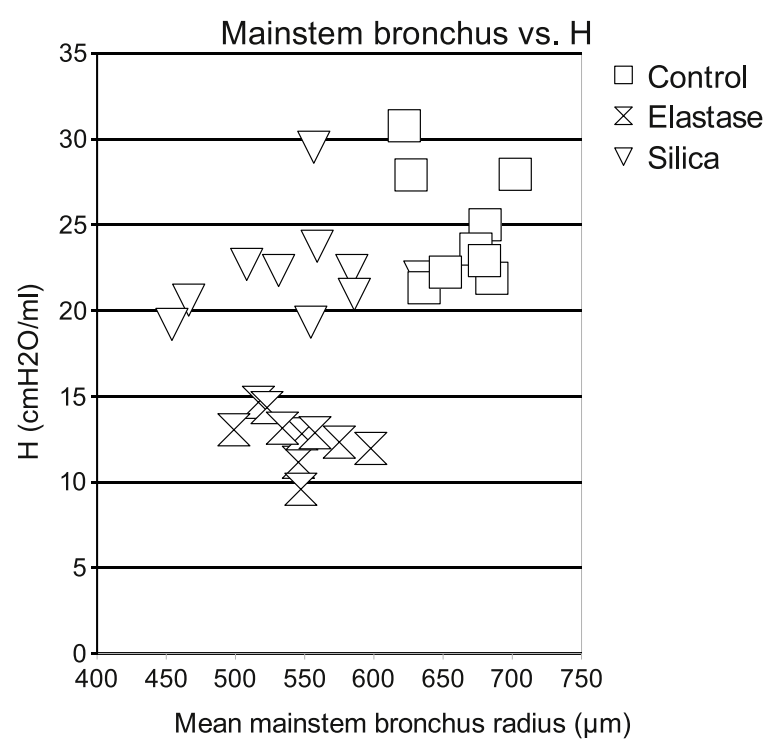

Figure 5. Mean radius of left and right mainstem bronchi plotted along with tissue elastance $(\mathrm{H})$.

Table 5. The effect of decreasing dose on the automatic segmentation. Mean TPVF and FPVF of the automatic segmentations with different levels of $\mathrm{x}$-ray dose, with the standard deviation in parentheses.

\begin{tabular}{lcll}
\hline & Dose 69.1 cGy & Dose 48.3 cGy & Dose 30.2 cGy \\
\hline TPVF (\%) & $86.59(6.02)$ & $82.39(11.01)$ & $84.15(2.88)$ \\
FPVF (\%) & $9.19(2.84)$ & $15.29(8.61)$ & $16.94(7.88)$ \\
\hline
\end{tabular}

Regarding the constant phase model parameters, Raw, $I$ and $G$ did not show any difference between the control and the diseased mice. $H$ was slightly lower in silica $(22.26 \pm$ $\left.2.94 \mathrm{cmH}_{2} \mathrm{O} \mathrm{ml}^{-1}\right)$ compared to control mice $\left(24.84 \pm 3.31 \mathrm{cmH}_{2} \mathrm{O} \mathrm{ml}^{-1}\right)$ and significantly lower in elastase-treated mice $\left(12.59 \pm 1.42 \mathrm{cmH}_{2} \mathrm{O} \mathrm{ml}{ }^{-1}\right)$. If mean mainstem bronchi radii and $H$ are plotted jointly, clear differences between the three groups can be seen (see figure 5). Silicotic mice have smaller bronchi radii than controls, and virtually the same tissue elastance. On the other hand, emphysematous mice also show smaller bronchi radii, but a significantly lower tissue elastance. This suggests that the causes of mainstem bronchi shrinking are different. In the inflammation case, the inflammation around the main airways is the most probable cause of radius reduction. In contrast, the reduced airway diameter in emphysematous mice is likely to be due to the increased compliance of their lung tissue, revealed by the low $H$ value.

Table 5 shows the mean TPVF and FPVF values for the images acquired at different $\mathrm{X}$-ray doses (see subsection 3.5). It can be seen that, as expected, decreasing dose leads to worse segmentations. However, the decrease in accuracy is not dramatic and, depending on the application, the algorithm might be of use even with very low dose images.

\section{Discussion and conclusions}

Accurately segmenting mice airways in in vivo micro-CT images is a challenging task. On the one hand, the imaging technology imposes certain image quality constraints, as mentioned 
above (see section 1). On the other hand, the position of lung structures can vary during the scanning process, which takes several minutes. Despite artificial ventilation, iso-pressure apneae and deep anesthesia, the way in which lungs respond to repeated mechanical ventilation does not remain constant during the acquisition process. We observed that this was especially significant in emphysematous specimens, which lead to a generally low image quality due to movement artifacts.

To overcome the difficulty imposed by the low SNR and movement artifacts, a robust segmentation algorithm is required. We built on a previously published framework for tubular structure segmentation and reconstruction. Previous works have centered on the framework itself and its applications (Schlathoelter et al 2002, Bülow et al 2004, van Ginneken et al 2008). The framework provides with generic concepts for the segmentation and reconstruction of treelike structures, such as the propagating wavefront and the segment definition. Here, we have used these basic concepts of the framework and we have devised new features in its different components, such as the variable threshold and the voting segment evaluation, to adapt to the particularities of thoracic micro-CT images. These extra components have certain tunable parameters that must be fixed. In our experience, it is useful to divide these parameters in wavefront propagation and segment evaluation parameters to better understand their global effect. To fine tune the parameters, a few images of the dataset should be used as a guide, until an acceptable trade-off between sensitivity and specificity is achieved.

We tested our algorithm on normal mice and on two mouse models of lung disease. The three groups showed very different image characteristics. Particularly, the elastase group displayed very low voxel intensity in the lungs, which made both manual and automatic segmentations more difficult. Moreover, movement artifacts were stronger in this group. Therefore, we used a different parameter set for this image dataset. The main characteristic of this parameter set was that it was more restrictive in both wavefront propagation and segment acceptance.

The validation was performed by comparing automatic segmentations with manual ones. In an attempt to quantify the existing human observer errors, a second expert segmented 50\% of the evaluated images. Results show that a considerable variability exists between the two observers, suggesting the difficulty of precisely delineating the airways.

The automated image analysis tool that we have developed may be of use for a variety of studies related to lung physiology and pathology. A number of potential applications of murine airway segmentation have been outlined in the introductory section. We plan to use this airway segmentation method to quantify both emphysema and inflammation, in the context of chronic-inflammation-related lung carcinogenesis. An interesting topic for future work would be the measurement of airways walls since they are also affected in COPD.

We showed that differences in the airway diameter among different groups can be detected with this segmentation method. However, the question of how early in a disease process changes can be quantified has not been addressed. A different dataset showing disease progression would be required. The factors that will affect the technique's sensitivity include image resolution, signal-to-noise ratio and the presence of motion artifacts in the images.

We observed that, despite the segment evaluation rules, some leaks remained undetected. Depending on the application, an interactive tool for incorrect segment removal might be of use. The relatively large standard deviations in the TPVF and FPVF values reveal that the algorithm performs better in some images than in others. This could be improved by modifying the tunable parameters separately for each image, which should be done automatically if a fully automatic algorithm is desired. Variables such as the number of segmented branches or the intensity values within and outside the already segmented airways could be used for an iterative tuning process. Another limitation of the algorithm is due to the fact that it is 
based on a propagating wavefront. Any interruption in an airway branch caused by noise or disease stops the propagation. Additional features would be required to detect and avoid these interruptions.

Segment hierarchical information is given together with the segmentation. Thus, the topology of the tree can be easily traced because the parent and children of each branch are identified. We believe that this is a major advantage of the technique when compared to other algorithms that simply segment the tree, for two reasons. First, topological information can be used to help the segmentation, for instance by comparing the current segment with its ancestors' radius or by checking the number of neighbor segments. Second, it eliminates the need for an extra skeletonization step. Moreover, our algorithm provides with measurements of the length, volume and mean wavefront size of each airway (which is a surrogate of the area). We showed an application of this by comparing the mean radius of the left and right mainstem bronchi among the control, emphysema and inflammation groups. Combining data from the constant phase model parameters and the airway measurements, we were able to detect different patterns in the different groups. These differences could not be clearly seen when only input impedance parameters or mainstem bronchi measurements were analyzed.

In conclusion, we have presented a fast and robust algorithm for murine airway segmentation and reconstruction. By adjusting the algorithm parameters to the particular characteristics of different models of lung disease, the algorithm is able to segment the airways with high sensitivity and specificity values. We have shown that measurements derived from these segmentations can be combined with pulmonary input impedance measurements to gain more insight into the changes in normal lung physiology caused by different diseases.

\section{Acknowledgments}

This research project was funded by the Spanish Ministry of Health (project FIS-PI070751), the Government of Navarra, the Spanish Ministry of Science and Innovation (Project MICCIN PSS-010000-2008-2) and the 'UTE Project CIMA'. X Artaechevarria is funded by a predoctoral grant of the Basque Government. A Muñoz-Barrutia holds a Ramón y Cajal Fellowship of the Spanish Ministry of Science and Innovation. C Ortiz-de-Solórzano was supported in part by the Spanish Ministry of Science and Innovation (MICCIN PSS010000-2008-2), the EU Marie Curie Program (MIRG-CT-2005-028342), and a Ramón y Cajal Fellowship.

\section{References}

Allen G B, Suratt B T, Rinaldi L, Petty J M and Bates J H T 2006 Choosing the frequency of deep inflation in mice: balancing recruitment against ventilator-induced lung injury Am. J. Physiol. Lung Cell. Mol. Physiol. 291 710-7

Aykac D, Hoffman E, McLennan G and Reinhardt J 2003 Segmentation and analysis of the human airway tree from three-dimensional X-ray CT images IEEE Trans. Med. Imaging 22 940-50

Bribiesca E 2008 An easy measure of compactness for 2D and 3D shapes Pattern Recognit. 41 543-54

Bülow T, Lorenz C and Renisch S 2004 A general framework for tree segmentation and reconstruction from medical volume data Medical Image Computing and Computer-Assisted Intervention 'MICCAI 2004' vol 3216 pp 533-40

Cassidy A, Duffy S W, Myles J P, Liloglou T and Field J K 2007 Lung cancer risk prediction: a tool for early detection Int. J. Cancer 120 1-6

Chaturvedi A and Lee Z 2005 Three-dimensional segmentation and skeletonization to build an airway tree data structure for small animals Phys. Med. Biol. 50 1405-19

Collins R A, Ikegami M, Korfhagen T R, Whitsett J A and Sly P D 2003 In vivo measurements of changes in respiratory mechanics with age in mice deficient in surfactant protein D Pediatr. Res. 53 463-7 
De Clerck N, Meurrens K, Weiler H, Dyck D V, Greet G V, Terpstra P and Postnov A 2004 High-resolution X-ray microtomography for the detection of lung tumors in living mice Neoplasia 6 374-9

de Torres J P et al 2007 Assessing the relationship between lung cancer risk and emphysema detected on low-dose CT of the chest Chest 132 1932-8

Deschamps T and Cohen L D 2001 Fast extraction of minimal paths in 3D images and applications to virtual endoscopy Med. Image Anal. 5 281-99

Dreyfuss D and Saumon G 1998 Ventilator-induced lung injury. Lessons from experimental studies Am. J. Respir. Crit. Care Med. 157 294-323

Fetita C I, Preteux F, Beigleman-Aubry C and Grenier P 2004 Pulmonary airways: 3-D reconstruction from multislice CT and clinical investigation IEEE Trans. Med. Imaging 23 1353-64

Ford N L, Martin E L, Lewis J F, Veldhuizen R A W, Drangova M and Holdsworth D W 2007 In vivo characterization of lung morphology and function in anesthesized free-breathing mice using micro-computed tomography J. Appl. Physiol. 102 2046-55

Ford N L, Thornton M M and Holdsworth D W 2003 Fundamental image quality limits for microcomputed tomography in small animals Med. Phys. 30 2869-77

Froese A R, Ask K, Labiris R, Farncombe T, Warburton D, Inman M D, Gauldie J and Kolb M 2007 Three-dimensional computed tomography imaging in an animal model of emphysema Eur. Respiratory J. 30 1082-9

Graham M W, Gibbs J D and Higgins W E 2008 Robust system for human airway-tree segmentation Proc. SPIE 6914 $1-18$

Hantos Z, Daróczy B, Suki B, Nagy S and Fredberg J J 1992 Input impedance and peripheral inhomogeneity of dog lungs J. Appl. Physiol. 72 168-78

Houghton A M, Mouded M and Shapiro S D 2008 Common origins of lung cancer and COPD Nat. Med. $141023-4$

Hu S, Hoffman E and Reinhardt J 2001 Automatic lung segmentation for accurate quantitation of volumetric X-ray CT images IEEE Trans. Med. Imaging 20 490-8

Ibanez L, Schroeder W, Ng L and Cates J 2003 The ITK Software Guide 1st edn (Kitware, Inc.) http://www.itk.org/ItkSoftwareGuide.pdf

Johnson K A 2007 Imaging techniques for small animal imaging models of pulmonary disease: micro-CT Toxicol. Pathol. 35 59-64

Kiraly A P, Higgins W E, McLennan G, Hoffman E A and Reinhardt J M 2002 Three-dimensional human airway segmentation methods for clinical virtual bronchoscopy Acad. Radiol. 9 1153-68

Lundblad L K A, Thompson-Figueroa J, Allen G B, Rinaldi L, Norton R J, Irvin C G and Bates J H 2007 Airways hyperresponsiveness in allergically inflamed mice: the role of airway closure Am. J. Respir. Crit. Care Med. 175 768-74

Namati E, Chon D, Thiesse J, Hoffman E A, de Ryk J, Ross A and McLennan G 2006 In vivo micro-CT lung imaging via a computer-controlled intermittent iso-pressure breath hold (IIBH) technique Phys. Med. Biol. 51 6061-75

Postnov A A, Muerrens K, Weiler H, van Dyck D, Xu H, Terpstra P and de Clerck N M 2005 In vivo assessment of emphysema in mice by high resolution X-ray microtomography J. Microsc. 220 70-5

Saffiotti U, Williams A, Daniel L, Kaighn M, Mao Y and Shi X 1996 Carcinogenesis by crystalline silica: animal, cellular, and molecular studies Silica and Silica-Induced Lung Diseases (Boca Raton, FL: CRC Press)

Schlathoelter T, Lorenz C, Carlsen I C, Renisch S and Deschamps T 2002 Simultaneous segmentation and tree reconstruction of the airways for virtual bronchoscopy Proc. SPIE 4684 103-13

Shi L, Thiesse J, McLennan G, Hoffman E A and Reinhardt J M 2007 Three-dimensional murine airway segmentation in micro-CT images Proc. SPIE 6511651105

Tomioka S, Bates J H T and Irvin C G 2002 Airway and tissue mechanics in a murine model of asthma: alverolar capsule vs forced oscillations J. Appl. Physiol. 93 263-70

Tschirren J, Hoffman E, McLennan G and Sonka M 2005 Intrathoracic airway trees: segmentation and airway morphology analysis from low-dose CT scans IEEE Trans. Med. Imaging 24 1529-39

Valentine R, Rucker R B, Chrisp C E and Fisher G L 1983 Morphological and biochemical features of elastase-induced emphysema in strain A/J mice Toxicol. Appl. Pharmacol. 68 451-61

van Ginneken B, Baggerman W and van Rikxoort E M 2008 Robust segmentation and anatomical labeling of the airway tree from thoracic CT scans Medical Image Computing and Computer-Assisted Intervention 'MICCAI 2008' pp 219-26

Vincent L 1993 Morphological grayscale reconstruction in image analysis: applications and efficient algorithms IEEE Trans. Image Process. 2 176-201

Wilcoxon F 1945 Individual comparisons by ranking methods Biometrics 1 80-3 